\title{
Макєঠovıкá
}

Tóp. 12 (1972)

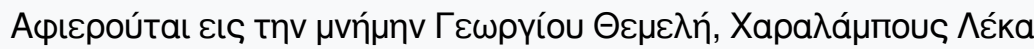

MAKGAONIKA

¿YГTPAMMA IIEPIOAIKON

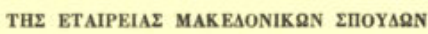

EIIMEAEIA,

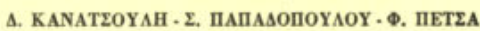

TOMOE $\triangle Q \triangle E K A T O \Sigma$

1972

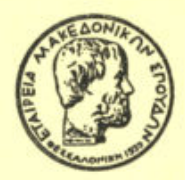

EN $\theta$ EIZAAONIKH

1972

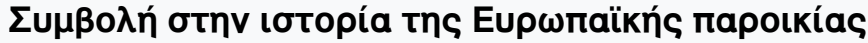

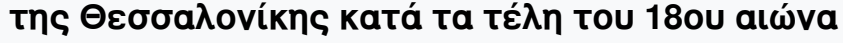

Constantin A. Vacalopoulos

doi: $10.12681 /$ makedonika.1004

Copyright ( 2015 , Constantin A. Vacalopoulos

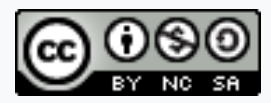

Aঠ¿ıа Xpńбnৎ Creative Commons Attribution-NonCommercial-ShareAlike 4.0.

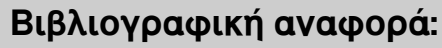

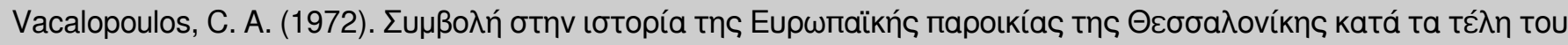
18ou aı́́va. Макєঠovıкá, 12, 183-200. https://doi.org/10.12681/makedonika.1004 


\section{CONTRIBUTION À L'HISTOIRE DE LA COLONIE EUROPÉENNE DE THESSALONIQUE VERS LA FIN DU XVIIIe SIĖCLE}

Un ancien professeur, qui avait enseigné au gymnase gréco-français de «Saint Jean-Baptiste De La Salle» et qui désire rester anonyme, m'a remis avec beaucoup de gentillesse la copie d'un manuscrit français, qui contient l'histoire d'Urendjick (résidence de vacances de la colonie européenne vers la fin du XVIIIe siècle), où se trouve la propriété des Frères. L'original, selon les renseignements du professeur, a été écrit par le frère Rodriguez dans un cahier à petits carreaux, de petites dimensions, un habituel cahier d'arithmétique, vers le début du XXe siècle. Il semble que c'est alors que fut composée l'histoire d' Urendjick.

Le Frère Rodriguez de la "Congrégation des Frères des Écoles Chrétiennes» naquit le 18 Février 1855 à la Chapelle-Aux-Bois du département Doubs. En 1888, il fut nommé professeur de français au gymnase gréco-français «De La Salle», de Thessalonique (Rue Franque, 10) où il enseigna avec zèle pendant cinquante quatre ans. Le gouvernement français, reconnaissant les services rendus aux écoliers par l'humble et affable moine, lui décerna le 5 Février 1938 la médaille de Chevalier de la Légion d'Honneur. Plus tard il fut honoré aussi des Palmes Académiques.

Vers l'année 1942, il rentra définitivement en France, où il mourut trois ans plus tard.

Le texte français est le suivant:

$$
\text { Historique d' Urendjick }
$$

composé par le frère Rodriquez, professeur du Collège De la Salle, Rue Franque 10.

Vers 1825, Djeck Abott, Grec de Religion, Anglais de Nation, eut l'idée de faire le commerce des sangsues, car à cette époqe la médecine en faisait un fréquent usage; celles de Hortiach, montagne de $1.200 \mathrm{~m}$ d'altitude, à 20 kilomètres de Salonique, étaient renommées dans tout l'Orient.

Au moyen de réclames, Abott fit connaître ces sangsues en Europe, après quelques mois les commandes affluèrent, Djeck en expédiait des milliers chaque jour: le prix augmenta avec la réputation. Après une année Abott vendait les sangsues Ifr. pièce. Au moyen d'un bon pourboire le rusé Salonicien monopolisa ce commerce si lucratif. 
p. 2 Des chevaux lancés dans les étangs où pullulaient ces vers aquatiques, revenaient couverts de ces annélides convoités; après quelques années le rusé commerçant possédait une fortune colossale... Mais ce qui vient par la flûte s'en va par le tambour.

Abott se retire des affaires et devient prodigue. Le vallon d'Azvesto-Kori= village de la chaux, s'étend de Hortiach à Platanakia. Dieck avait tant de fois admiré ce superbe panorama, du sommet de la montagne, qu'il résolut de l'immortaliser... Abott chaisit le centre de ce vallon inculte, pittoresque, mais sale... ce lieu se nommait Eurumedjeck=araignées; Djeck acheta pour quelques livres turques se repaire d'insectes couvert de rochers escarpés, d'une étendue de 20 hectares, au moins; après une transformation féérique ce lieu fut appelé: "Urendjick=petit paradis). Tous les richards de Salonique firent construire une villa dans ce vallon fortuné près d'Abntt!

p. 3 Le nouveau prodigue met 600 ouvriers à l'auvre: les mineurs firent sauter les rochers à la dynamite...les pierres servirent à bâtir le château, les murs d'enceinte, les jardins en terasses... d'autres ouvriers transportaient la terre de la plaine à dos de mulets... plusieurs des meilleurs jardiniers de la ville, organisèrent les parterres; les plus savants arboriculteurs commandèrent des arbres rares des pays éloignés: plaqueminiers et bignoniers d'Amérique; cèdres et pins du Liban, figuiers et vignes de Smyrne...

Après cinq ans de travaux et un million de livres turques (22fr. 50$)$ dépensé, la transformation était complète.

p. 4 Les consuls étaient invités à passer les vacances à Urendjick; Monsieur Cousinéry Consul de France, intime ami de Djeck, a consigné dans un magnifique ouvrage en deux volumes, la joyeuse vie que menaient là, ces Messieurs, à cette époque peu reculée et qui cependant paraît mythologique. Le service religieux était en honneur; tous les dimanches un prêtre catholique célebrait la Messe, dans la chapelle dont les ruines se voient encore aujourd'hui; le chant des fidèles s' unissait au bruit des cascades et au chant des oiseaux; ensuite les boissons étaient servies sur la place à l'ombre des cèdres et des marronniers; au milieu, un jet d'eau de vingt mètres de hauteur, entouré des statues qui ornent le jardin de la Banque ottomane actuelle, car là était la maison natale de Djeck Abbot.

p. 5 En 1858, le Sultan Abdoul-Medjid, visita Salonique et fut logé à la maison de Djeck aujourd'hui Banque ottomane; Abdoul-Hamid, fis du Sultan, accompagnait son père; il était âgé de 12 ans: il ne supposait pas alors qu'un demi-siècle plus tard il reviendrait à Salonique en captivité.

Ces deux illustres personnages furent invités par le propriétaire à visiter Urendjick...ce que fut accepté avec grand plaisir. Alors Abbot fit élargir le sentier qui conduisait à cette superbe villa, fit construire des ponts, acheta tous les tapis turcs qui se trouvaient dans les magasins et en couvrit la route depuis la maison 
de Djeck jusqu'à Urendjick, c'est-à-dire sur une longueur de sept kilomètres.

Un bain turc fut construit exprès pour leurs Majestés, il coûta 25.000 francs, il nous sert actuellement de chapelle. Enfin le jour fixé pour cette visite arrive: Tout Salonique est en fête, les chorales, les fanfares, les orchestres, précedent, accompagnent, suivent le cortège... les voitures de luxe roulent sur les tapis... au milieu du parcours, la Soeur de Morel, Supèrieure de l'Orphelinat de Zéitenlick s'avance une requête à la main...Abdoul Medjid fait arrêter les voitures, lit la lettre, écrit au crayon: "Accordé à perpértuité». Il met sa signature. C'était une prise d'eau gratuite pour son Etablissement que recevait Soeur de Morel.

Après quelques instants le Sultan se trouve en face du superbe portrait d'Urendjick; il pose la jambe droite sur le marchepied...

Mais le ciel s'était couvert, un éclair éblouissant, suivi d'un coup de tonnerre épouvantable, furent regardés comme mauvais augure et sa Majesté remit la jambe droite près de la gauche et refusa de descendre...

Quelle déception pour Djeck! Il était à l'apogée de sa richesse, de sa prodigalité et de sa gloire; mais la roche Tarpéienne était près du Capitole aussi pour Abott... Il pria le Sultan d'accepter un café: le mangal (réchaud) doré est apporté; le liquide est chauffé avec des billets de banque pour une valeur de 60.000 francs... Le Sultan demanda au Maréchal qui l'accompagnait si le café est meilleur chauffé avec le papier quavec le charbon...

En ce moment Djeck apporte les clefs de la propriété sur un plateau d'argent et les offre aux illustres Visiteurs; le Padischa les tourne, les retourne, puis les rend à Abott en lui disant "Votre belle propriété sera exempte d'impôts tant que vous serez possesseur).

Les fils du prodigue voyant leur fortune se fondre, prirent la direction des affaires... leur père honteux, garde la chambre et se laisse mourir de faim de peur d'être empoisonné par ses fils. On ne peut pas graver sur sa tombe l'épitaphe de l'Avare: "Ci-gît un homme qui s'est laissé mourir de faim pour conserver de quoi vivre).

Les deux fils se firent un procès au sujet de la maison paternelle que chacun revendiquait; l'aîné fit venir le bâtonnier des avocats de Paris: le cadet invita le bâtonnier des avocats de Londres; le procès dura longtemps... les plaideurs malvus à Salonique, depuis la mort du père, vendirent les propriétés qui leur restaient et se retirèrent sans tambour ni trompette en Angleterre.

Voilà ce que j'ai lu ou entendu raconter par des témoins oculaires, sur Urendjick, aujourd'hui campagne des Frères. Voici un fait qui s'y rapporte directement et dont j'ai été témoin puisque je suis ici depuis quarante ans.

Vers 1870, Urendjick devint propriété du Baron Frédéric de Charneaud, qui enleva les marbres et les jeunes arbres rares pour embellir ses maisons et jar- 
dins du quartier franc et de Calamari; le Petit Paradis tomba peu à peu à l'état où nous le voyons aujourd'hui.

En 1895, Mr Edouard de Charneaud, vendit ces ruines à raison de 200 napolèons, à Mr Pascal Ruggiéro, qui les répara un peu, construisit un pont sur le torrent et céda cette propriété aux Frères, en 1902, pour moins de 10.000 francs. Un petit-neveu de Djeck était revenu à Salonique, il se fixa au bord de la mer, se livra au commerce et acquit le vaste terrain qui porte aujourd'hui le nom d' Abott! Son fils Robert fut enlevé en 1907, par une bande de 15 brigands de Kiretch-Keui, voici comment.

Jusqu'au 20 e siècle, les bandes de voleurs enlevaient les riches habitants raïas, c'est-à-dire sujets européens, ils faisaient payer une forte rançon, ordinairement 15.000 livres turques $=22 \mathrm{fr} 50$; le Consul du captif obtenait la somme exigée, du Sultan, par l'ambassadeur!

p.11 Or en 1907 se forma la dernière bande à Kiretsch-Keui; leur victime fut Robert Abott, arrière-petit-neveu de Djeck; il fut arrêté à minuit, sur le seuil de sa porte: bailloné, les yeux bandés, il est porté par deux membres de la bande tirés au sort, sur la colline; de là on le fait marcher jusqu'au bain d'Urendjick où il passe la première muit, puis la 2 e dans le moulin à vent, puis 40 jours dans une masure abandonnée, hors du village.

Pendant ces 42 jours Robert fut fort bien soigné; mais sa famille était angoissé. Le Consul d'Angleterre remue ciel et terre pour éclaircir le mystère, la police cherche, cherche encore, cherche toujours; rien, encore, rien, toujours rien! Après une semaine, le chef de bande envoie un de ses hommes masqué, porter ces deux

p. 12 mots au Consul d'Angleterre: "Robert Abott est entre nos mains, bien portant, très bien soigné; mais si vous arrêtez le porteur, si vous avertissez la police, nous vous enverrons la tête du captif; que votre ambassadeur fasse payer 15.000 livres turques au Sultan, alors nous rendrons Robert).

Ainsi fut fait: la Banque ottomane versa 8.000 livres et la Banque de Thessalonique le reste, la somme fut portée à minuit, l'heure des crimes! Les livres furent comptées, vérifiées et partagées par les 15 brigands masqués; alors la vue et la liberté furent rendues à la victime, qui arriva à l'aube à la maison, après 42 jours d'absence!!!

Quelles fêtes après de telles craintes! Le héros fut visité, interrogé nuit et jour; il ne savait ni qui l'avait arrêté ni où il était resté caché durant six semaines.

p. 13 Les policiers, les gendarmes, les soldats à pied, à cheval, battaient la campagne, fouillaient au loin... les brigands étaient tout près et fort tranquilles. Trois membres de la bande plus rusés que les autres partirent le soir même pour Volo, avec leurs mille livres turques chacun; ils depensèrent joyeusement leur argent durant trois ans, après quoi ils furent saisis par la police; les 12 autres furent pris sur place en 1907 durant les vacances voici comment: 
Le 29 Juin, jour de fête, les coupables étaient réunis dans le grand café du village, ce fut leur malheur: "Toujours par quelque endroit les fourbes sont pris». Une femme fâchée, accuse un des bandits... Kémal bey, mon ancien élève, capitaine de gendarmerie, se trouvait là... Quelle chance pour lui: la femme en colère lui donne les noms des 15 coupables; deux jours après les douze étaient en prison et Kémal bey était nommé Commandant et décoré... Après des tortures de jour et de nuit Nicolas et Athanase, fils de Papas, avouèrent tout...alors les menottes aux mains, les détenus conduits par douze gendarmes arrivent à Urendjick et au village pour indiquer où l'argent était caché. Le Gouvernement rentra ainsi en possession de 12.000 livres turques. Les criminels jugés furent condamnés à 5 ans, 10 ans et 15 ans de prison... Mais en 1908, grâce à la nouvelle Constitution, tous les captifs furent mis en liberté. Je pourrais vous citer 25 histoires parfaitement authentiques, dont j'ai été le témoin, toutes dans ce genre.

Principaux arbres d'Urendjick:

Io Cèdres, 20 Mûriers, 30 Pistachiers, 4o Figuiers, 50 Pommiers, 60 Poiriers, 70 Pruniers, 80 Amandiers. 9o Pins, 100 Chènes, 11 o Platanes, 120 Marronniers, 130 Ormes, 140 Plaqueminiers, 15o Cyprès, 160 Négondos, 17o Sophoras, 180 Kakis, 190 Arbousiers, 20o Cornouillers, etc...

Comme nous voyons, Urendjick, était la résidence de vacances des commerçants européens de Thessalonique. Il se trouve dans un beau vallon, sur la route qui conduit à Asvestohori et sur laquelle, vers 1926, un peu à l'ouest de la propriété des Frères de la Congrégation de Saint Français De La Salle (figure 1) s'installèrent cinq ou six familles d'Asie Mineure occidentale et cinq de Thrace ${ }^{1}$. Jusqu'à ces dernières années on pouvait voir des ruines de constructions dans la région d'Urendjick, deux sources, ainsi que la propriété des frères, conservée relativement en bon état.

La résidence d'été des Européens, selon les renseignements de Rodriguez, que nous allons vérifier plus bas, était la création de Djeck Abbot, commerçant Anglais de nationalité, mais orthodoxe grec de religion, qui vers 1825 eut l'idée de faire le commerce des sangsues si avantageux à cette époque. Au sujet de la famille Abbot, un raport vénitien de 1792 nous apprend que le commerçant anglais Barthélemy Edouard Abbot, installé à Thessalonique, remplaçait le consul Vénitien ${ }^{2}$. Quant à savoir quel est le rapport entre Barthélemy Edouard Abbot et le Djeck du manuscrit, nous l'apprenons par le premier tome du livre

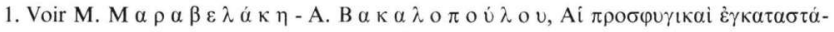

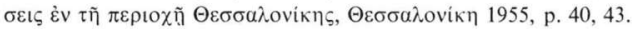

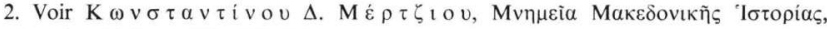

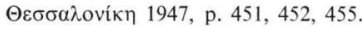


de la vieille thessalonicienne Angélique B. Metallinou (Tsiomou), Vieille Thessalonique-Thessalonique illustrée, qui n'a pas d'indication chronologique, mais qui doit avoir été édité autour de 1936-1940. D'après l'auteur de ce livre, Barthélemy Edouard Abbot était le chef de la famille Abbot. Jeune homme, il vint de Constatinople et s'installa à Thessalonique vers 1771. Le fils d'Edouard

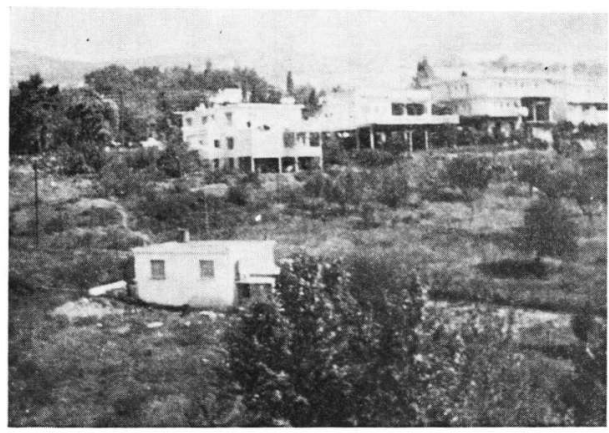

Fig. I. Vue d'une partie d'Urendjik

était Frédéric Abbot et les enfants de celui-ci Robert (connu comme Babis), celui que nous connaissons par le manuscrit Djeck (Djékis), Nelson et d'autres $^{1}$. Metallinou insére même le portrait de Barthélemy Abbot (figure 2) après celui de Djeck Frédéric Abbot (figure 3), ainsi que d'autres membres de la famille Abbot ${ }^{2}$.

La famille Abbot possédait à part la propriété d'Urendjick, un manoir renommé (un château, comme on appelait alors les maisons de campagne) au bord de la mer, à peu près à l'endroit où se trouve l'actuelle station d'autobus, Misrahi. Mais il semble qu'il vivait pendant l'hiver à Salonique même dans une maison, appartenant à Jean Gouta Kaftantzoglou, parce que (avec la maison de Charnaud $^{3}$ de qui nous allons parler plus bas) elle est mentionnée dans son testament du 27 Décembre $1817^{4}$.

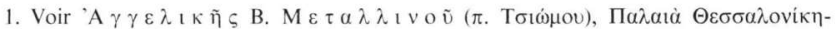

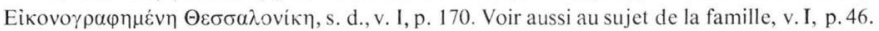

2. $M \varepsilon \tau \alpha \lambda \lambda \downarrow v \circ \tilde{v}$, ibid., s. I, p. 170, 171, 172-181.

3. E. M i 11 e r, Le Mont Athos, Vatopèdi et l'ile de Thasos, Paris 1889, p. 280, mentionne une dame Charnot et il dit qu'elle appartenait à une des plus grandes familles de Cavala.

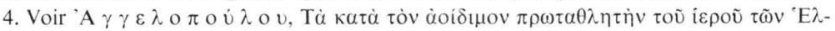

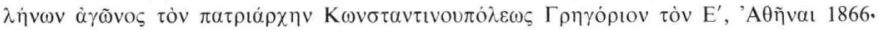
v. I, p. 421. 
Urendjick avait acquis renommée assez grande pour être mentionné jusque dans le livre du celèbre géographe italien Andrien Valvi ${ }^{1}$, traduit de l'italien en grec et édité à Vienne vers 1839 par l'érudit de Larissa Constantin Koumas. Un siècle plus tard, le géographe allemand Leonhard-Shultze Jena, se fondant, sans doute sur des témoignages écrits, dit que les consuls d'Allemagne, d'Angle-

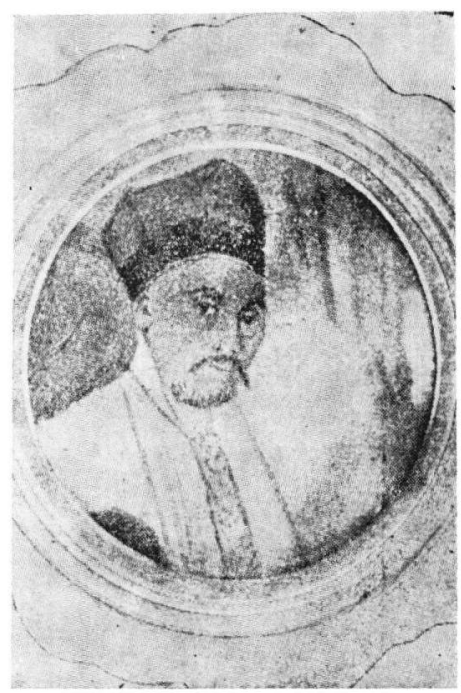

Fig. 2. Barthélemy Edouard Abbot

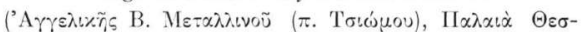

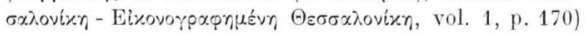

terre, de France, de Venise, de Naples, de Raguse, de Hollande, du Danemark et de Suède avaient des maisons de campagne particulières à Urendjick et ils avaient formé une sorte de communauté unie (einträchtige Gemeinde)2.

Nous trouvons la première mention de la résidence d'été des commerçants européens avec une brève description de la région chez le voyageur anglais Ro-

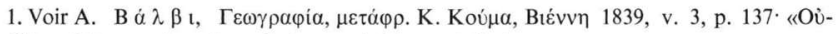

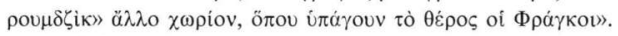

2. Voir L e o n h a r d - S h u ! t z e J e n a, Makedonien, Landschafts-und Kulturbilder, Jena 1927 , p. 221. 
bert Walpole, qui y passa le 18 Août $1801^{1}$. Mais c'est le voyageur anglais Henry Holland, de passage à Thessalonique vers 1812 ou 1813, lorsque Charnaud y était consul d'Angleterre, qui nous apprend ce qu'était cette communauté unie des Européens de Salonique et comment elle vivait alors. Charnaud appartenait à une famille levantine, tandis que sa femme était de Hollande. Elle-même

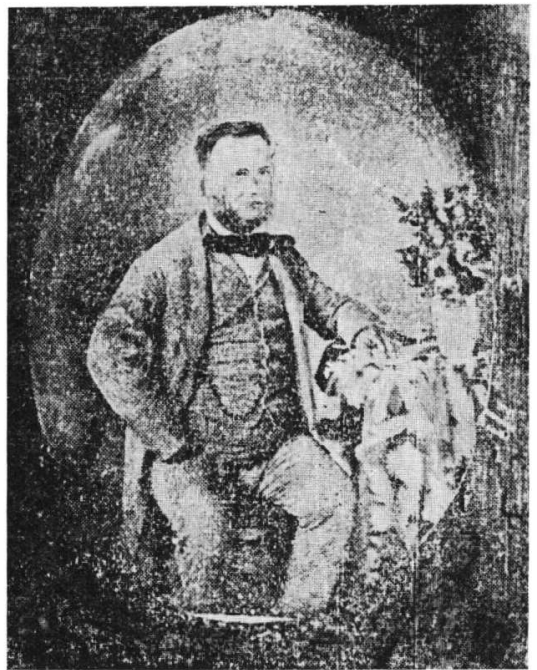

Fig. 3. Djeck Abbot

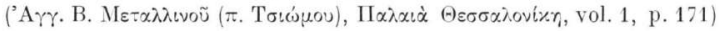

et ses filles n'avaient jamais quitté la Turquie et elles ne connaissaient pas très bien l'anglais. Ce fait, ainsi que leur mode de vie oriental, qui était celui de presque tous les occidentaux qui habitaient à Salonique contribua à leur faire perdre quelques-unes de leurs caractéristiques nationales ${ }^{2}$.

Charnaud était hospitalier envers tout compatriote de passage et il avait même soin de le présenter à d'autres amis, européens de marque. Il présent ainsi le voyageur Clarke, de passage à Thessalonique vers 1805 ou 1806 à un

1. Voir Robert Wal pol e, Memoirs relating to European and Asiatic Turkey and other countries of the east, éd. 2e, London 1818, v. I, p. 273.

2. Voir $\mathrm{H}$ en r y $\mathrm{H}$ oll an d, Travels in the Ionian Isles, Albania, Thessaly, Macedonia etc., During the years 1812 and 1813, London 1815, p. 312. 
«Gentleman» nommé Abbot, que l'on appelait le père de Levant Company. Celui-ci, selon moi, doit être Barthélemy Abbot, lui même. Clarke nous dit encore qu'Abbot avait un frère qui possédait une maison de commerce à Constantinople, qu'il l'avait connue et plusieurs fois visitée ${ }^{1}$.

Nous complétons ces renseignements par les témoignages de Holland, d'après lesquels Abbot était un commerçant anglais de Thessalonique, qui s'y était installé depuis près de 42 ans, après avoir passé 13 ans au nord de l'Asie Mineure (à Proussa ou à Nicomédée semble-t-il où il était peut-être le représentant de son frère qui avait une maison de commerce à Constantinople). Il avait épousé une Grecque d'Asie Mineure, et lui-même ne parlait pas bien l' anglais. Il portait les habits du pays et parlait le turc, à peu près comme sa langue maternelle. Il fréquentait surtout les Turcs et un étranger pouvait le prendre pour un Turc. Le seul qui parlât anglais dans la famille était son fils, Frédéric Abbot, qui avait épousé une Anglaise. Les coutumes grecques de cette famille, surtout celles des dames et des demoiselles qui pratiquaient pointilleusement les jeûnes de l'Eglise orthodoxe avaient frappé Holland².

D’après ce dernier, le chef de la famille, Barthélemy Edouard Abbot, se serait installé à Thessalonique vers 1771, date qui correspond exactement à celle que nous fournit Metallinou ${ }^{3}$.

Des renseignements très intéressants sur les membres féminins de la famille d'Edouard Barthélemy Abbot nous sont aussi donnés par Clarke, dont il a été question ci-dessus et qui avait été charmé par leur amabilité. Les femmes assises à l'orientale, sur le divan, parlaient avec vivacité et étaient curieuses de connaître quel était le but du voyage de l'Anglais et quelles impressions il en avait tirées. Elles racontaient encore différentes anecdotes sur leurs amis et connaissances communes de Constantinople. Leur compagnie réjouit Clarke et les remercia de leur bonne atmosphère qu'elles avaient creée dans une ville que l'on avait décrite comme foyer d'épidémies ${ }^{4}$.

Cependant non seulement les Abbot, mais aussi les autres étrangers qui formaient la colonie des occidentaux à Thessalonique avaient subi intensément l'influence du milieu grec, comme par exemple, le consul autrichien, ainsi qu'un certain monsieur Chassaud. Ce Chassaud tient certainement son origine d'un autre, Jean Chassaud, qui était venu de Marseille et s'était installé comme boulanger à Thessalonique dès le début du XVIIIe siècle. Il y était venu avec sa

1. Voir Da n i e l E dwa r d C la r k e, Travels in Various Countries of Europe, Asia and Africa, London 1816, Part the Second, Section the Third, p. 346.

2. Holl a nd, ibidem, p. 313.

3. Voir $\mathrm{M} \varepsilon \tau \alpha \lambda \lambda \imath \vee$ ṽ, ibid., p. 170.

4. Voir C la r k e, ibid., p 347. 
famille: en effet le registre des baptêmes de l'église catholique de Thessalonique nous apprend que sa fille Marianne était née à Marseille vers 1771 ainsi que sa femme Claire Olive. À Thessalonique il eut cinq enfants ${ }^{1}$.

Donc le descendant probable de ce Jean Chassaud, ainsi que le consul autrichien avaient épousé des grecques et leurs filles, les plus cultivées des femmes de Thessalonique étaient plus grecques qu'européennes en ce qui concernait la langue, les manières et habillement. Ces demoiselles chantèrent un soir pour leurs invités étrangers des chants turcs et grecs et parmi ces chants, le chant patriotique de Rigas «Qu'attendez-vous amis et frères» qui était très populaire à cette époque. Ce fait est caractéristique aussi de l'atmosphère patriotique qui régnait parmi les membres grecs des familles occidentaux-levantines. La mélodie de ce chant rappelait à Holland le chant anglais «Life let us cherisch») ${ }^{2}$.

Des éléments complémentaires sur la société des européens de Thessalonique et surtout sur leur vie à la résidence d'été d'Urendjick nous sont donnés aussi par E. M. Cousinéry, ancien consul général de France (figure 4). Cousinéry fut nommé consul de France à Thessalonique le 27 Août 1786 avec un salaire mensuel de 9.000 livres et il arriva à son poste le 20 Août 1787. Dès lors il commença sa correspondance avec le Ministère de la Marine et la Chambre de Commerce de Marseille. Ses rapports se distinguent par l'abondance des informations et par les renseignements importants, qu'il donne sur la situation économique du pays ${ }^{3}$. En Mai 1792 Cousinéry épouse une grecque, malgré la désapprobation de sa famille et de ses supérieurs ${ }^{4}$. L'année suivante, le $16 \mathrm{Mars}$ 1793, Lebrun, ministre des Affaires Etrangères après des intrigues contre Cousinéry l'arrête de son poste et l'oblige de rentrer en France et de porter plainte au ministre des Affaires Etrangères. Finalement Cousinéry s'établit définitivement à Smyrne, où il s'adonne à ses chères études archéologiques ${ }^{5}$. Là, en Mai 1814 il apprend la nouvelle de la restauration des Bourbons et le 24 Septembre de la même année le Ministère des Affaires Etrangères le rétablit dans ses fonctions avec un salaire de 12.000 francs $^{6}$.

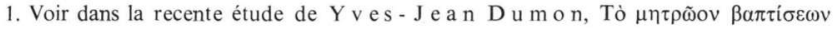

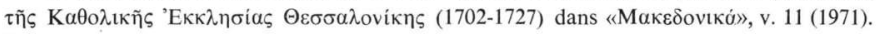
Voir page 45 et suivantes où il y a des renseignements sur les baptêmes des membres de la colonie catholique ainsi que sur les métiers qu'ils pratiquent (commerçants, boulangers, hôteliers, marins, tailleurs etc.).

2. Voir Holl and, ibid., p. 322, 323.

3. Voir Lou is Bergasse, Souvenirs de Marseille et des échelles du Levant au XVIIIe siècle, Marseille 1921, p. 33.
4. Ibid., p. 37-39.
5. Ibid., p. 40-57.
6. Ibid., p. 57-59. 
Tout ce que raconte Cousinéry dans son livre sur sa vie, les Européens, Thessalonique et les divertissements de leur société se rapporte à «l'âge d'or» comme il appelle la période précédant 1792, quand les événements politiques n'avaient pas encore troublé sensiblement ce port d'Orient. Les guerres napoléonniennes n'avaient pas encore commencé et il n'y avait pas de désaccords

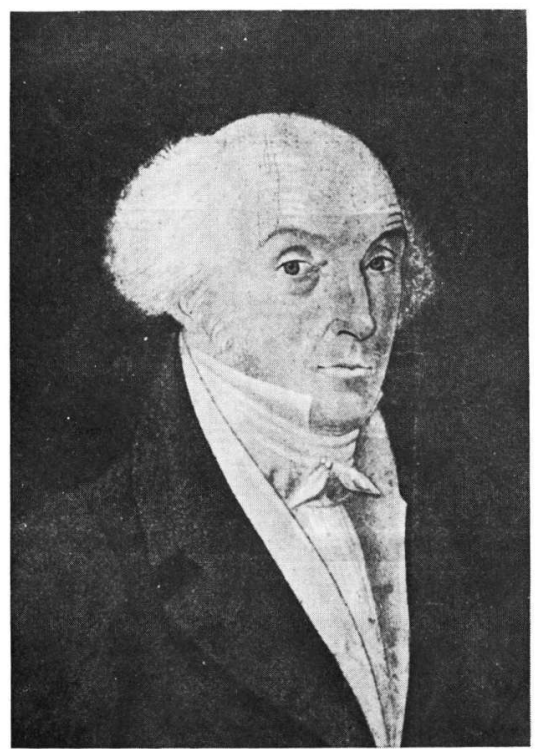

Fig. 4. E. M. Cousinéry

(Louis Bergasse, Souvenirs de Marseille et des Echelles du Levant au XVIIIe siècle, entre pages 32-33)

profonds entre les membres de la colonie française ${ }^{1}$. Il y avait même encore des relations amicales entre les différents commerçants européens qui étaient installés à Thessalonique.

Cousinéry raconte donc qu'une grande partie des commerçants européens de Thessalonique avait construit des villas dans le vallon d'Urendjick. Ces villas étaient entourées de jardins avec beaucoup de verdure en particulier des

1. Ibid., p. 40 . 
chênes et des platanes d'Italie. La vie de ce quartier était pittoresque. Chaque matin les commerçants européens partaient par groupes pour descendre à la ville, d'où, ils revenaient le soir. Leurs familles les attendaient sur les rochers entourés de gazon et là elles les recevaient et écoutaient les nouvelles du jour. Chaque soir ils se rassemblaient tous chez un consul ou un commerçant et ils dansaient. Quelquefois ils s'entendaient pour de petites excursions aux alentours.

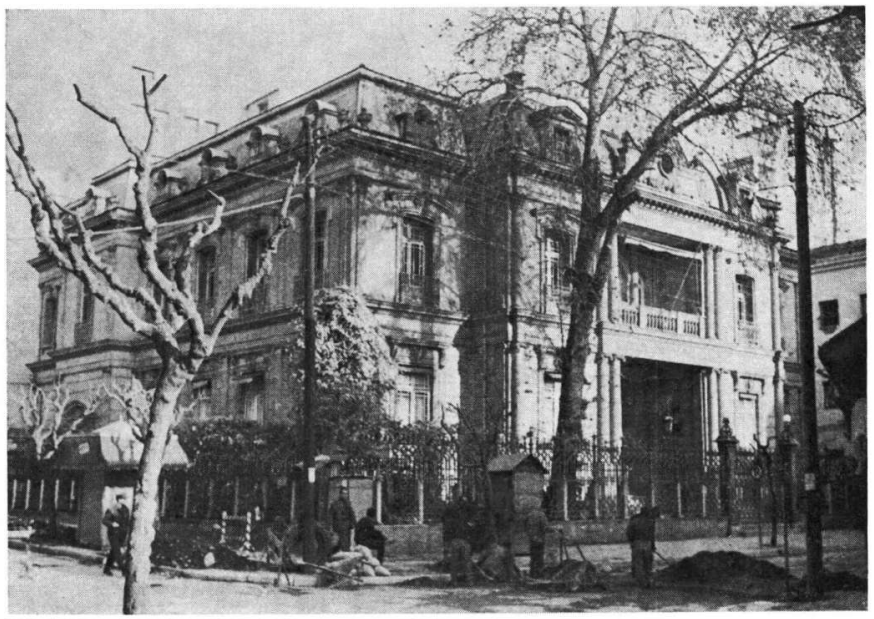

Fig. 5. La maison de Djeck Abbot. Aujourd'hui : les bureaux de I.K.A. (de la Sécurité Sociale)

Les distractions unissaient ces hommes que l'hostillité nationale et les intêrets économiques séparaient. Tel était «l'âge d'or» des commerçants occidentaux de Salonique, vers la fin du XVIIIe siècle.

Avec la déclaration des guerres napoléoniennes (1792-1814) et le bouleversement général dans toute l'Europe cette vie insouciante fut interrompue pour de nombreuses années. Quand le consul général de France E. M. Cousinéry revint à son poste après la restauration, c'est à dire après 1814 , ce paysage idyllique avait complétement changé de forme. Les belles maisons de campagne n'étaient plus que ruines et n'étaient habitées que par les hiboux, comme il dit de façon caractéristique. Leurs anciens propriétaires ne s'étaient point soucié de les réparer et elles s'écroulaient ${ }^{1}$. Le quartier d'été des commerçants euro-

1. Voir E. M. Co u s i né ry, Voyage dans la Macédoine, Paris 1831, v. I, p. 107-109. 
péens qui avait été négligé, n'accomplissait donc plus sa destination d'antan.

Les renseignements ci-dessus nous permettent de conclure que Djeck Abbot n'était point celui qui avait pris l'initiative de choisir le lieu d'Urendjick ni celui qui avait bâti la première villa vers 1825 , comme le rapporte le Frère Rodriguez dans sa narration historique. En 1825, comme nous avons vu, cette

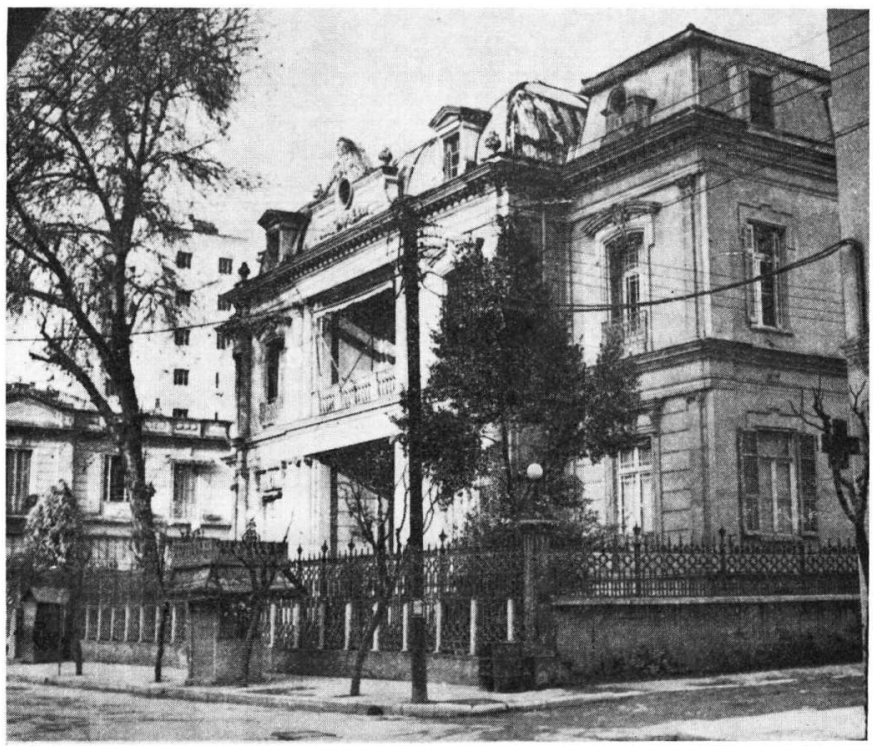

Fig. 6. La maison de Djeck Abbot

région de délassement des Européens avait perdu pour toujours sa beauté. Il semble que Djeck Abbot soit l'unique de tous les étrangers qui conserva sa villa consulaire jusqu'au XXe siècle, de sorte qu'il y reçut le sultan AbdoulMedzid en 1858, quand celui-ci visita Thessalonique et s'y présenta comme prince réformateur, supprimant les distinctions entre les musulmans et les non musulmans ${ }^{1}$.

C'était l'époque de Tanzimat, après la fin de la guerre de Crimée. Le sultan fut alors logé à Thessalonique chez Djeck, dont la maison est encore là

1. Voir P. R is a l, La ville convoitée Salonique, Paris 1917, p. 240. 
de nos jours: elle se trouve sur la Rue Franques, c'est le bâtiment connu comme Banque Othomane et qui abrite aujourd'hui les bureaux de I.K.A. (de la Sécurité Sociale) (figures 5 et 6 ).

Les souvenirs récents qu'avaient les Thessaloniciens de la visite du sultan ainsi que le fait que cette propriété ait été durant longtemps la possession de
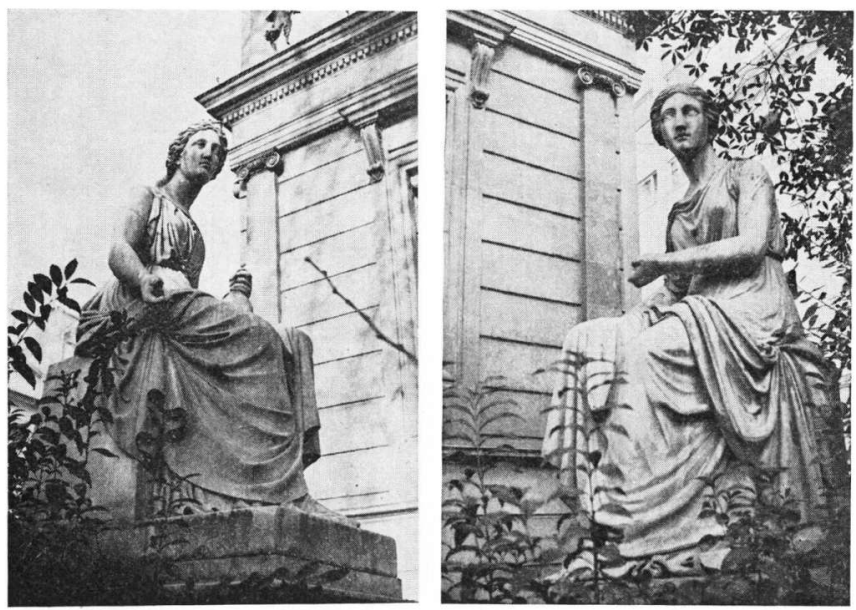

Figures 7-8. Les deux statues de femmes, qui se trouvent dans le jardin du bâtiment des bureaux de I.K.A.

Djeck, fit penser à tort que celui-ci avait été le premier à bâtir sa villa à Urendjick. En réalité le fondateur du domaine était son grand-père Barthélemy Edouard Abbot. Mais si les informations de frère Rodriguez au sujet de la création de la résidence des occidentaux ne sont pas justes, au contraire les autres, plus recentes, qui se rapportent à la diversité des arbres d'Urendjick (une grande partie d'entre eux sont rares et proviennent des pays lointains, comme p. ex. bignona et le plaqueminier d'Amérique), à l'histoire des membres de la famille Abbot, à la reception du sultan et aux privilèges que celui-ci a accordé au propriétaire sont intéressantes et dignes de foi. Nous apprenons encore que les deux statues de femmes (figures 7 et 8 ) qui se trouvent maintenant dans le jardin du bâtiment des bureaux de I.K.A. ornaier.t auparavant le domaine des Abbot à Urendjick. Nous ne savons pas quand exactement elles ont été transportées du domaine à la maison paternelle des Abbot de la rue Franque; peut- 


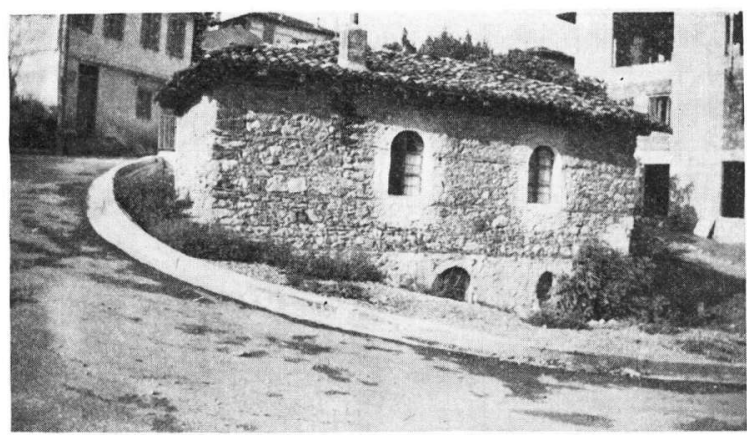

Fig. 9. La plus vieille maison d'Asvestohori

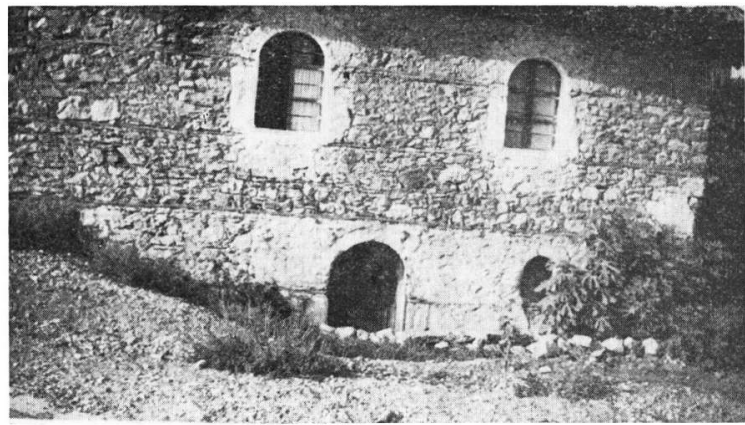

Fig. 10. La maison d'Asvestohori vue du côté septentrional

être après la visite d'Abdoul-Medzid à la villa en 1858. Quelles personnes représentent-elles ces statues? Ce sont des figures féminines, assises pendantes. Elles portent un habit et une tunique. Leur cou long, leur tour de tête, leur taille assez haut ceintée et en général la partie mince du haut de la taille par rapport à la partie accentuée du bas de la taille, permettent de supposer que l'original lointain de ces statues contemporaines devait être la statue d'une Muse ou d'une Nymphe des temps alexandrins, comme a eu la bonté de me faire connaître le professeur, Monsieur Démétre Pandermalis. L'une d'elles, celle de gauche en rentrant à la villa, tient à la main gauche une bourse.

En 1870, Fréderic de Charnaud (sans doute descendant du consul anglais 
Charnaud, mentionné, comme nous l'avons vu, par Holland dans son livre) acheta le domaine de Djeck. Frédéric de Charnaud enleva les marbres et les rares arbres du domaine pour embellir les maisons et les jardins qu'il possédait dans le quartier des occidentaux et dans la campagne du Salonique, au-delà de la Tour Blanche.

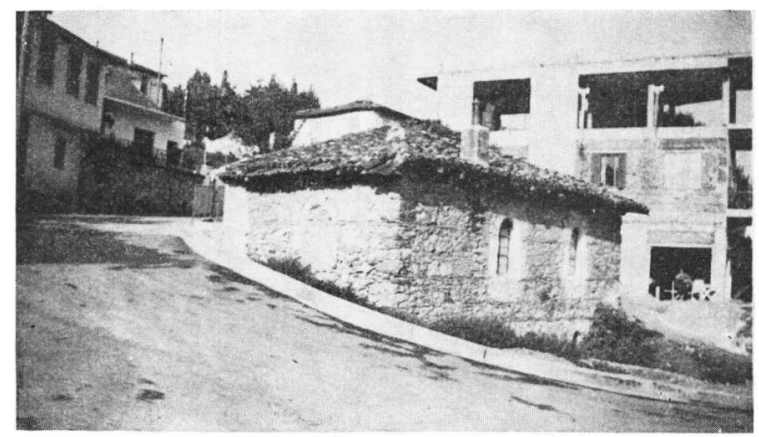

Fig. 11. La maison d'Asvestohori vue du NE

Tout ce que rapporte le Frère Rodriguez sur le manque de sécurité dans la campagne de Macédoine et hors de la ville même est aussi très intéressant. Rappelons aussi le cas de la capture de Robert Abbot, petit neveu de Djeck, par quinzaine de bandits, habitants d'Asvestohori vers 1907. Frère Rodriguez raconte avec beaucoup de détails cet événement dont rien n'est dit dans le livre de Charilaos G. Tsekos, «Histoire d'Asvestohori», Salonique 1957. Tout ce que rapporte le frère Rodriguez complète donc le chapitre relatif à la question «Mauvaise administration et manque de sécurité» pages 56-57 du livre cité.

Tsekos (p. 38-40) nous parle aussi de l'installation des Agrafiotes (habitants de Agrafa) dès 1812 à l'endroit appelé jusqu' aujourd-hui Vlachicos Lakkos, où se trouve l'actuelle rivière d'Asvestohori qui a été comblée ces mois derniers pour des raisons d'hygiène. Cependant cette installation est ancienne, antérieure à $1600^{1}$, comme le prouvent les documents tures relatifs à la question.

La maison représentée ici (figures 9, 10, 11) est sans doute l'une des plus anciennes; je l'ai remarquée et photographiée. Elle est unique, me semble-til, dans le village aujourd'hui.

CONSTANTIN A. VACALOPOULOS

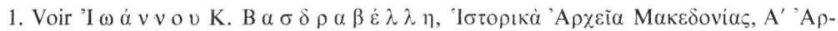

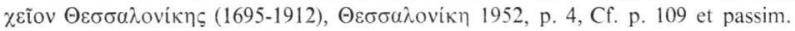




\section{ПЕРІ}

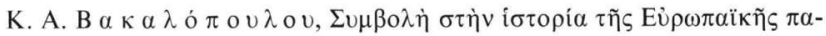

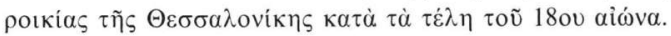

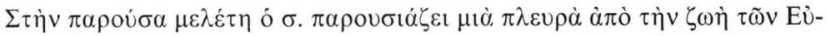

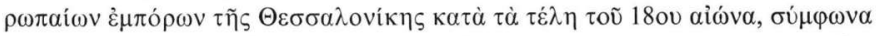

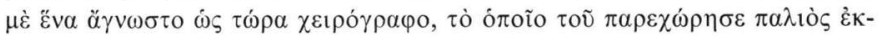

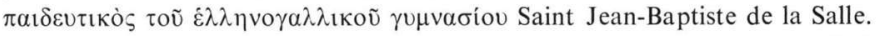

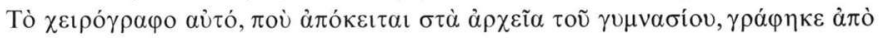

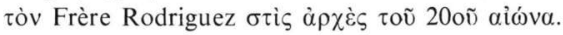

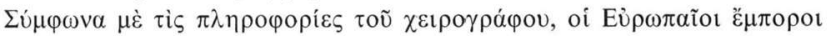

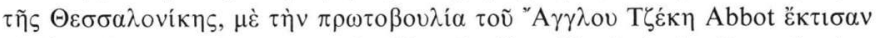

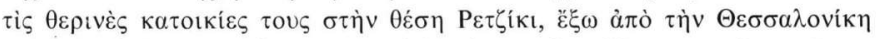

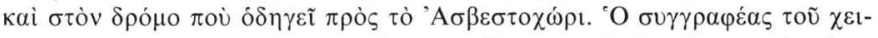

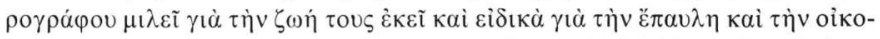

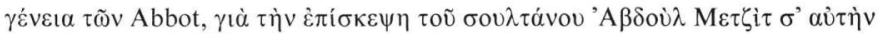

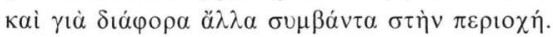

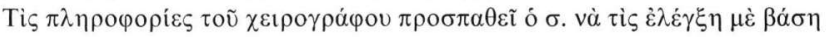

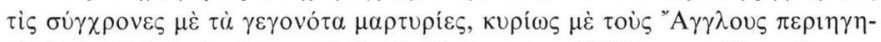

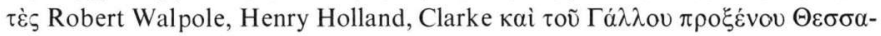

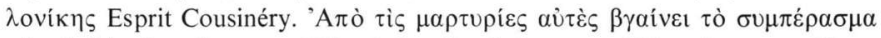

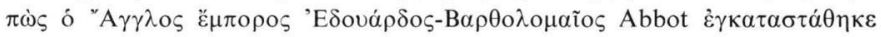

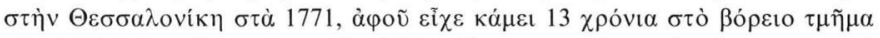

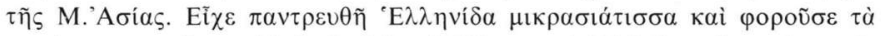

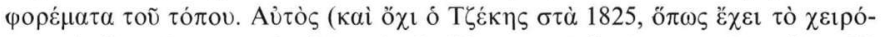

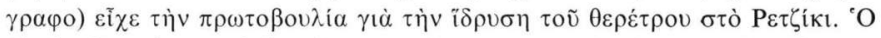

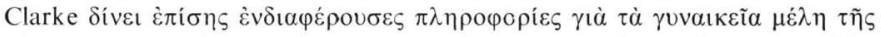

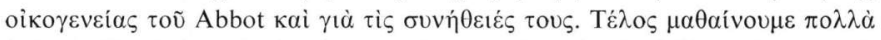

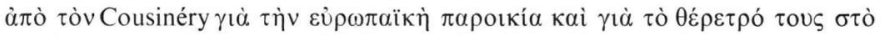

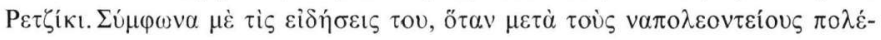

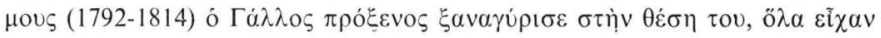

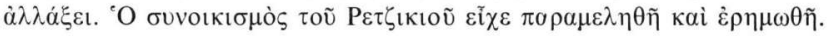

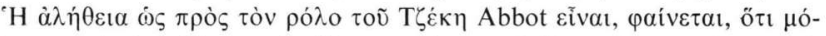

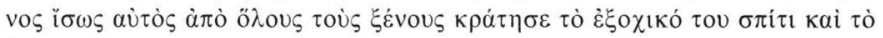

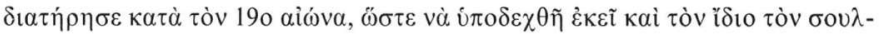




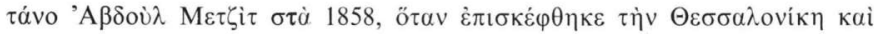

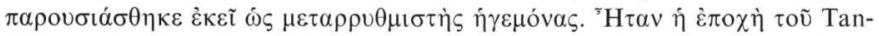

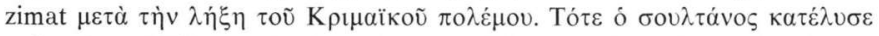

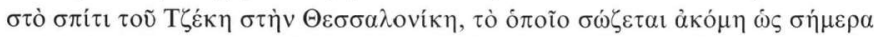

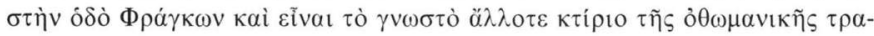

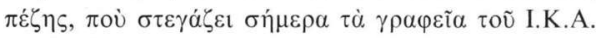

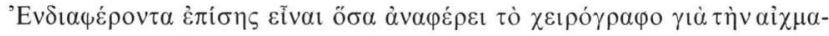

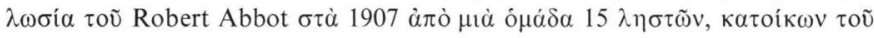

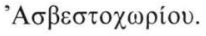

